\title{
Editorial \\ Welcome to Magnetism: A New Open Access Scientific Journal on Magnetism, Magnetic Materials and Magnetic Technology
}

\author{
Gerardo F. Goya

Magnetism has been entangled with human progress since the first realization by Chinese civilization of the attractive interactions between loadstone and iron, which they attributed to a similar $q i$ (vital force). From the first compass that helped sailors to navigate the oceans, through the first electromagnetic machine or magnetic data storage devices, to the present awesome advances in magnetic materials and devices, magnetism has been deep-rooted in human history and is ingrained into our current everyday life. Over the centuries, we have expanded and deepened our understanding of the fundamentals of magnetic phenomena and their technological tricks. Our current knowledge has allowed producing great hallmarks in the fields of spin-based logic, giant magnetoresistance sensors and quantum spin Hall low-dissipation spintronics, among others. Meanwhile, advances in nanoscience and biomedicine have created a niche where magnetism plays a key role by allowing diagnosis and therapies, including magnetic resonance imaging; remotely actuated nanosystems for drug release; cell actuation and magnetically induced cell ablation in oncology.

The above series of advancements in magnetism and magnetic materials makes it difficult to predict limits for what can be achieved in the future. The new journal Magnetism (ISSN 2673-8724) is aimed to provide a platform for the scientific community to share these new advancements and a forum for active exchange of new results and ideas. Magnetism is brought to this arena aiming to disseminate high-quality research through the publication of both papers and review articles addressing innovative theoretical and experimental results on magnetism, magnetic materials and magnetic technology. We welcome researchers worldwide to contribute to the Magnetism journal in any emerging or consolidated topics of magnetism, including, but not limited to, the following:

- Spintronics;

- $\quad$ Magnetism of magnetic interfaces;

- Magnetization dynamics, spin waves and magnonics;

- Biomagnetism;

- $\quad$ Magnetically driven diagnostics tools;

- Magnetic therapies in biomedicine;

- Micromagnetics;

- Nanomagnetism;

- Magnetic thin films, interfaces and multilayers;

- $\quad$ Strongly correlated electrons systems (SCES);

- $\quad$ Spin-transfer-based phenomena and devices;

- Magnetic information storage and MRAM;

- $\quad$ Electromagnetic waves and energy;

- Magnetic materials and technologies;

- Magnetic-based metrology tools and techniques.

The progress in many areas of magnetism also requires close interaction with many neighbor scientific disciplines, and therefore the cross-fertilization among these disciplines is of paramount importance to shift the current state of the art. As such, the Magnetism 
journal expects to be of significance for any member of the scientific community enthusiastic to participate in this project.

I look forward to counting on your participation in this exciting journal.

Gerardo F. Goya, PhD

Editor-in-Chief for Magnetism

Conflicts of Interest: The author declares no conflict of interest.

\section{Short Biography of Author}

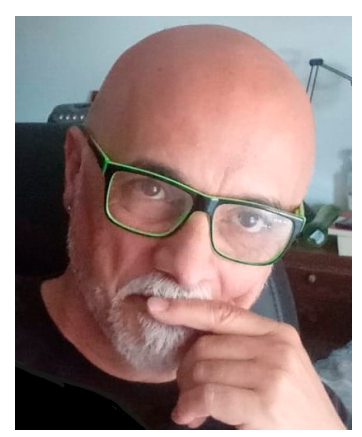

Prof. Gerardo F. Goya (Mar del Plata, Argentina) obtained his PhD degree from the Universidad Nacional de La Plata, Argentina, in 1995. From 2001 to 2007, Prof. Goya was associate professor at the Institute of Physics, University of Sao Paulo (Brazil), where he created and managed the mechanochemistry laboratory at the Materials Physics Department (DFMT). Prof. Goya was awarded a Ramon y Cajal Grant for incorporation of talented researchers and joined the Institute for Nanoscience and Materials of Aragon (INMA) (https://inma.unizar-csic.es/) at the University of Zaragoza, Spain, where he started and consolidated a new research line on biomedical applications of nanomagnetism and magnetic hyperthermia. Since 2012, he has been an associate professor at the University of Zaragoza and a full-time senior researcher, as well as co-founder and scientific advisor of nB Nanoscale Biomagnetics S.L., a spin-off company from UNIZAR that develops instrumentation for the biomedicine field. He has more than 170 international publications (h-index $=42$ ) with more than 7100 citations, 2 PCT patents and more than 120 conference presentations including more than 60 invited and keynote talks, as well as many short courses for diverse target audiences. 